\title{
In situ characterization of block copolymer ordering on chemically nanopatterned surfaces by time-resolved small angle $x$-ray scattering
}

\author{
K. O. Stuen, C. Liu, A. M. Welander, G. Liu, J. J. de Pablo, and P. F. Nealey ${ }^{\text {a) }}$ \\ Department of Chemical and Biological Engineering, University of Wisconsin, Madison, Wisconsin 53706 \\ D. K. Satapathy, K. Nygård, O. Bunk, H. H. Solak, and J. F. van der Veen \\ Department of Synchrotron Radiation and Nanotechnology, Paul Scherrer Institut, CH-5232 Villigen, \\ Switzerland
}

(Received 7 June 2008; accepted 2 September 2008; published 1 December 2008)

\begin{abstract}
The assembly of lamella-forming block copolymer blend thin films on chemically nanopatterned striped surfaces was monitored in real time with small angle x-ray scattering (SAXS) in transmission mode. The strongest diffraction from the assembled grating structure was detected after $4.5 \mathrm{~min}$ of annealing as the temperature was ramped from 100 to $240{ }^{\circ} \mathrm{C}$ at a rate of about $20^{\circ} \mathrm{C} / \mathrm{min}$. Real-space images were also obtained from samples annealed for specific times using top-down scanning electron microscopy (SEM) and this identified structures formed during annealing that are unique to the block copolymer blends. The data are compared to previously reported SEM and molecular simulation studies with pure block copolymers. Because it can be used in real time and probes the entire film thickness, transmission SAXS proved to be a useful tool for better understanding the block copolymer annealing process. (C) 2008 American Vacuum Society.
\end{abstract}

[DOI: $10.1116 / 1.2991977$ ]

\section{INTRODUCTION}

The directed assembly of block copolymer films on chemical patterns for nanolithographic patterning has the potential to address some of the grand challenges in lithography facing the semiconductor industry. ${ }^{1-10}$ In addition to offering a potential route to pattern smaller feature sizes, the insertion of the directed assembly of block copolymers into advanced lithographic processes (Fig. 1) may improve the pattern lineedge roughness (LER) ${ }^{1}$ and critical-dimension (CD) control. ${ }^{2}$ LER and CD control are recognized as the most significant current challenges for advanced lithography. ${ }^{3-5}$ For block copolymer lithography to be a viable lithographic option, however, it must be easily integrated into existing tools and procedures. ${ }^{11}$ Ideally, block copolymer spin coating and annealing must be optimized to be as simple and rapid as the current practice of spin coating and baking a photoresist. We previously showed that thin films of block copolymers can be annealed on a hot plate within a matter of minutes, suggesting that the block copolymers could satisfy the throughput requirements of the lithographic process. ${ }^{12}$ There is much more to be understood about block copolymer annealing, however, and such knowledge is necessary to engineer new processes that satisfy the requirements of advanced lithography while maintaining sufficiently rapid assembly.

Attempting to understand the block copolymer annealing process, others have studied block copolymer annealing in the bulk ${ }^{13-19}$ and in thin films on unpatterned ${ }^{20-24}$ and topographically patterned surfaces. ${ }^{25-28}$ The mechanisms described in these studies were based on the annihilation of defects through diffusion, which are generally slow to occur.

a) Author to whom correspondence should be addressed; electronic mail: nealey@engr.wisc.edu
In contrast, the mechanism for the directed assembly of lamella-forming block copolymers on chemical patterns is based on the propagation of order from the patterned surface through the film thickness. ${ }^{29,30}$ Since the directed assembly process usually involves thin films, below $100 \mathrm{~nm}$ in thickness, the annealing process is fast. The overall block copolymer grain size is dependent only on the size of the chemical pattern.

While it would be beneficial to monitor the threedimensional structure of the block copolymer annealing process over time, gathering this full spatial and temporal information is extremely difficult. Other groups have studied the internal structure of thin films of block copolymers using transmission electron microscopy, ${ }^{31} \mathrm{x}$-ray scattering, neutron scattering, or a combination of these techniques. ${ }^{32-34}$ For annealed films it is also possible to remove thin layers of the film to probe its internal structure, such as with a focused ion beam ${ }^{35}$ or mechanical slicing. ${ }^{36}$ To our knowledge, however, no previous study has been reported to probe block copolymer films in real time that are annealed in the presence of nanopatterned surfaces.

We report the use of small angle x-ray scattering (SAXS) in transmission mode to examine the mechanism and kinetics of the annealing of a block copolymer film on a chemical pattern. Transmission SAXS provides us with the capability to analyze the morphological development of the block copolymer in real time throughout the thickness of the film. We focus on the annealing process of a ternary blend of block copolymers and homopolymers to meet experimental constraints that require working with relatively large block copolymer domains (100 nm period) over areas $\sim 0.5 \mathrm{~mm}^{2}$. Ternary blends have been shown to be useful in the directed assembly of semiconductor fabric architectures. ${ }^{7,9}$ Combining the SAXS data with scanning electron microscope 


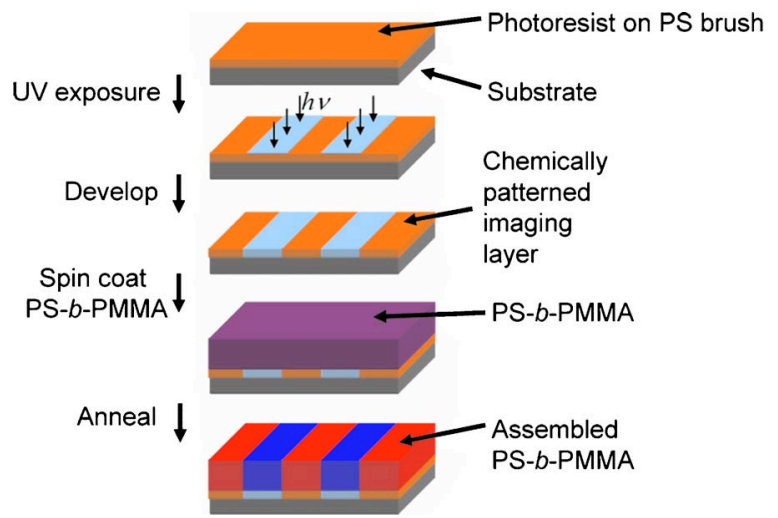

FIG. 1. Schematic of the directed assembly process on chemically nanopatterned substrates. A PS brush layer is grafted to the substrate, which is then coated with a photoresist. The photoresist is patterned using advanced lithography and etched, thereby transferring the pattern to the PS brush. A block copolymer film is spin coated onto the chemically patterned substrate and annealed, resulting in the assembled block copolymer.

(SEM) images, we have developed a better understanding of how assembled lamellae form from an initially disordered state in thin film ternary blends.

\section{EXPERIMENT}

\section{A. Materials}

Hydroxy-terminated polystyrene (PS) $\left(M_{n}=4 \mathrm{~kg} / \mathrm{mol}\right)$ was synthesized as reported previously using atom transfer radical polymerization ${ }^{37}$ with a 2-hydroxyethyl 2bromoisobutyrate initiator. Polymers for the block copolymer blend were purchased from Polymer Source, Inc. (Dorval, Quebec) and used as received. The blend in this study consisted of a lamella-forming polystyrene-block-poly(methyl methacrylate) block copolymer [PS- $b$-PMMA, $M_{n}=176$ $\mathrm{kg} / \mathrm{mol}$, polydispersity index $(\mathrm{PDI})=1.12]$ combined with homopolymers of PS $\left(M_{n}=77 \mathrm{~kg} / \mathrm{mol}, \mathrm{PDI}=1.05\right)$ and poly(methyl methacrylate) (PMMA) $\left(M_{n}=75 \mathrm{~kg} / \mathrm{mol}\right.$, PDI= 1.05). The ratio of the molecular weights of the homopolymers relative to the block copolymer, $\alpha$, was $\sim 0.43$. PS and PMMA were added in equal amounts to produce an overall volume fraction of the homopolymer in the blend, $\varphi$, of 0.24 . The Si wafers used in this experiment were $530 \mu \mathrm{m}$ thick.

\section{B. Sample preparation}

The general technique of the directed assembly of block copolymers on chemically patterned substrates is shown in Fig. 1 and described by Edwards et al. ${ }^{8}$ First, PS brushes were prepared by spin coating a thin film of hydroxyterminated PS on a $\mathrm{Si}$ substrate covered with a native oxide and then heating the film for $48 \mathrm{~h}$ at $160{ }^{\circ} \mathrm{C}$ to covalently bond the PS brushes to the native oxide. The treated Si substrate was then cleaned to remove unbonded PS, and subsequently an $~ 50$-nm-thick film of photoresist was spin coated on top of the brush. The photoresist was patterned using the X-ray interference lithography (XIL) facility at the Swiss Light Source. ${ }^{38}$ The exposed pattern consisted of lines and spaces with $100 \mathrm{~nm}$ period, $L_{S}$, over an area of 0.5 $\times 1 \mathrm{~mm}^{2}$. Such a large patterned area, relative to the pattern period, was required for the transmission SAXS experiment. After exposure and development of the photoresist, the patterned samples were exposed briefly to low power oxygen plasma. The oxygen plasma oxidized the exposed brush but did not remove it entirely. ${ }^{39}$ The photoresist was stripped from the substrate with chlorobenzene. The block copolymer film was then spin coated from a toluene solution to produce a film that was $\sim 24 \mathrm{~nm}$ thick. Separate samples were prepared for SAXS and SEM analysis.

\section{Sample characterization}

The block copolymer samples were characterized in situ by transmission SAXS and by SEM. SAXS was performed at the coherent small angle x-ray scattering (cSAXS) beamline at the Swiss Light Source. The cSAXS beamline had a number of features that produced sufficient signal and spatial resolution to carry out the experiment. The light source produced a bright x-ray beam and the fast, single-photon counting PILATUS detector collected high-resolution images. A $7 \mathrm{~m}$ flight tube helped improve the spatial resolution at the detector. The block copolymer samples were placed onto the beamline stage and annealed while scattering data were collected, enabling the scattering intensity to be collected as a function of scattering vector $q$ and time. The time between scans was $15 \mathrm{~s}$. The stage temperature was ramped from 100 to $240{ }^{\circ} \mathrm{C}$ with a ramp time of $\sim 6 \mathrm{~min}$ and held at $240{ }^{\circ} \mathrm{C}$ for an additional $20 \mathrm{~min}$.

Samples for SEM imaging were annealed on a hot plate for various times and then quenched to room temperature to arrest the assembly. The hot plate was ramped with a temperature profile similar to that employed at the cSAXS beamline. Top-down SEM images were acquired using a LEO1550 VP field-emission microscope using an accelerating voltage of $1 \mathrm{kV}$ and a $30 \mu \mathrm{m}$ aperture. Fourier transforms of the SEM images were generated to monitor the formation of the grating structure over time.

\section{RESULTS AND DISCUSSION}

Top-down SEM images for various annealing times are shown in Fig. 2. The sequence of SEM images of the surface of the blend annealed on chemical patterns shows the evolution of the nanostructures in the blend at the surface. Beside each image, a magnified version of its Fourier transform is shown. Immediately after spin coating ( $0 \mathrm{~min})$, small domains were visible, formed as the casting solvent evaporates from the film. These domains were randomly organized and varied in size. During annealing, the size of these domains increased and the domains oriented with the stripes of the chemical pattern (1.5 and $3 \mathrm{~min})$. The domains continued to coalesce into elongated structures that appeared on the surface to be tortuous and primarily randomly oriented (4.5 min). These domains likely consisted of lamellae oriented along the stripes at the chemical pattern interface, with bridge structures connecting one stripe to the next. After an additional $2.5 \mathrm{~min}$, the tortuous domains organized into well- 

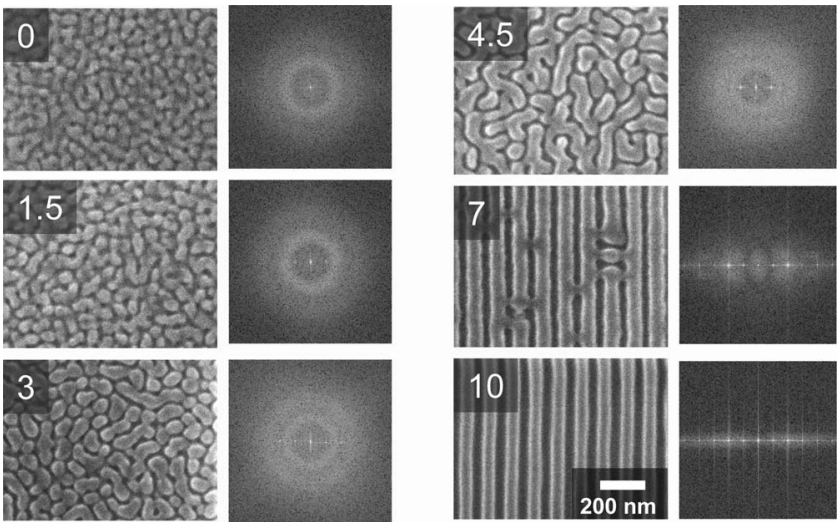

FIG. 2. Top-down SEM images of block copolymer films on chemically patterned surfaces with their magnified Fourier transforms. The numbers denote annealing times in minutes. The scale bar is $200 \mathrm{~nm}$ in length.

defined lamellae that were oriented with the chemical pattern (7 min) but still contained a number of pattern defects in the form of bridges between lamellar domains at or near the free surface of the film. These bridges were the last defects to be removed by the thermal annealing process. Finally, pattern perfection was achieved after $10 \mathrm{~min}$.

To monitor the formation of a structure in the film it is useful to consider the Fourier transforms (FTs) of the images shown in Fig. 2. Figure 3(b) shows the Fourier-space intensity as a function of wave vector taken along a horizontal line through the center of the Fourier transforms of Fig. 2. As the degree of ordering in the film at a certain period increases, the intensity of the peak in the intensity plot at the corresponding wave vector also increases. An increase in the number of peaks at regular intervals indicates a grating structure with sharper interfaces. From the sample annealed for 10 min, four peaks are visible that are multiples of the first order peak at $q=0.0063 \AA^{-1}$, clearly corresponding to the final grating structure. The first order grating diffraction peak appeared after $1.5 \mathrm{~min}$. At $3 \mathrm{~min}$, the ordering from the chemical pattern increased and the second order peak and a very faint third order peak appeared. The intensity of the second and third order peaks decreased at $4.5 \mathrm{~min}$, an indication of some temporary decrease in ordering when compared to the structure at $3 \mathrm{~min}$. After $7 \mathrm{~min}$ these peaks reappeared and remained strong in the final 10 min annealed structure. The FT analysis of SEM images is useful for following the formation of various structures at the top surface of the block copolymer blend film.

We can compare the top-down SEM images and their Fourier transforms with the SAXS data, which are de facto thickness averaged, to develop a more complete understanding of the block copolymer ordering mechanism. The SAXS diffraction intensity versus $q$ curves at various time points are displayed in Fig. 3(a). From this figure we can clearly see that the block copolymer domains produced a significant signal, which is remarkable considering that we are probing a $24-n m-t h i c k$ polymer film on a $530-\mu \mathrm{m}$-thick wafer with domains that have very similar refractive indices. This plot shows the diffraction intensity as a function of $q$ integrated (a)

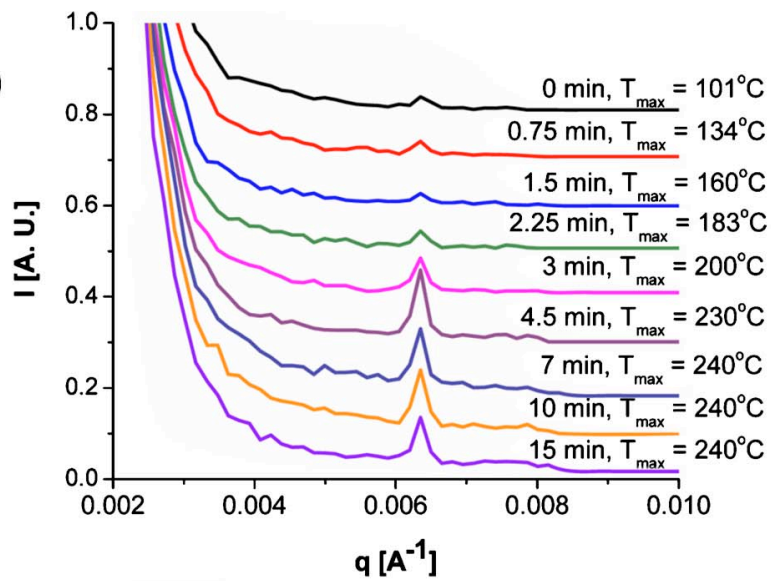

(b)

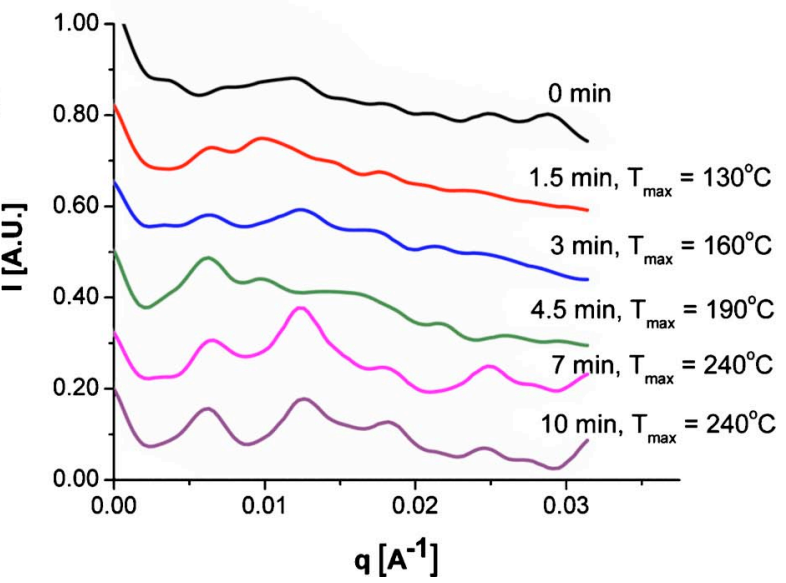

FIG. 3. (a) SAXS intensity vs $q$ for various annealing times. Intensity is given in arbitrary units. (b) Fourier intensity vs $q$ for the SEM images shown in Fig. 2. $T_{\max }$ is the highest temperature the sample reached at the time the measurement was taken.

over a narrow stripe in the direction of the grating diffraction peaks. After $10 \mathrm{~min}$, the peak at $0.0063 \AA^{-1}$ was fully developed, corresponding to the assembly of block copolymer domains registered to the stripes of the chemical pattern with a period of $100 \mathrm{~nm}$. Initially, this peak was much smaller but still present, indicating that the grating structure formed by the block copolymer near the substrate immediately after spin coating was detectable by the technique. The peak continued to increase in intensity after $2 \mathrm{~min}$ and reached a maximum at $4.5 \mathrm{~min}$, showing the rate at which the block copolymer domains align with the substrate pattern. After $4.5 \mathrm{~min}$ the peak intensity decreased and this may be due to degradation of the block copolymer from x-ray damage or thermal oxidation. Analysis of these mechanisms is beyond the scope of this study.

Edwards $e t a l .{ }^{29}$ previously studied the mechanism of ordering of pure block copolymers on chemically patterned surfaces. Through a combination of experiments and molecular simulations they demonstrated that the annealing takes place through various stages. This process is depicted schematically in Fig. 4. Initially a thin layer of block copolymer wets the chemically patterned substrate, with each block wetting the portion of the chemical pattern with which it has a 
(a)

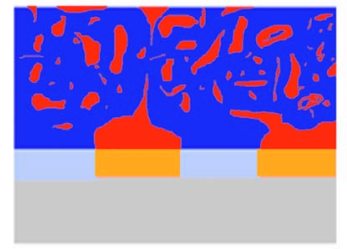

(b)

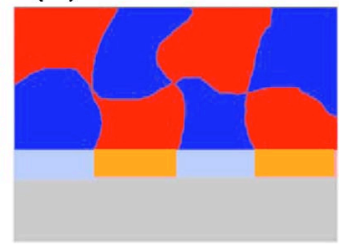

(c)

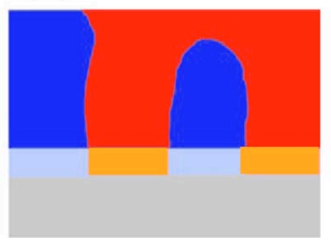

(d)

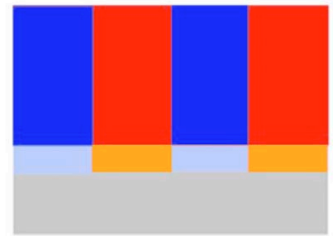

FIG. 4. Schematic of block copolymer film cross section during annealing based on the results of Edwards et al. The PS and PMMA domains are dark gray (red online) and black (blue online), respectively, and the chemical surface pattern is light gray (light blue online) and medium gray (orange online). (a) Initial state of block copolymer film after spin coating. (b) After a short annealing time each block copolymer block wets the patterned region with a similar surface energy and the opposite block is forced to be directly above that region. (c) Substrate pattern order propagates through the film, leaving bridge structures near the free surface. (d) Final equilibrium morphology of lamellae assembled on the striped chemical pattern.

similar surface energy [Fig. 4(a)]. After a short time of annealing, the block copolymer forms spot structures near the free surface of the film that are positioned in rows aligned with the chemical pattern. As annealing progresses, the spot structures coalesce into linear domains that at the free surface are generally offset from the chemical surface pattern by one-half of the pattern period $\left(L_{s} / 2\right)$. These structures vary throughout the film thickness, as shown in Fig. 4(b). Because of the orientation of the block copolymer chains, these structures are in some ways more similar to cylinders oriented parallel to the substrate than lamellae oriented perpendicular to the substrate. These structures are also similar to those that can form when $L_{S}$ is greater than the natural repeat of the block copolymer, $L_{0} \cdot{ }^{39}$ Further annealing leads to the propagation of order from the substrate to the free surface of the film, resulting in lamellae oriented perpendicularly to the substrate with few, if any, defects and long-range order.

Our results from SAXS and SEM with the block copolymer blend generally match the process described above, although a few differences exist. Edwards et al. did not find a tortuous lamella-like structure shown at $4.5 \mathrm{~min}$ or the types of bridge structures shown at $7 \mathrm{~min}$. The differences with the work by Edwards et al. may be related to the use of the blend in our work or the differences in the molecular weights of the block copolymers. The distribution of homopolymer in the direction perpendicular to the substrate may not be uniform and these blend structures could be partly influenced by the preferential segregation of the homopolymer to the free surface.
Using SAXS, we detected the largest signal from the first order diffraction peak after 4.5 min of annealing, indicating a high degree of order after this time. The SEM images taken at $4.5 \mathrm{~min}$, however, indicate a low degree of order at this time point. This apparent discrepancy can be understood by considering the different probing depths of SAXS and topdown SEM. Because the assembly process involves the propagation of order from the substrate to the surface, the free surface is necessarily the last part of the film to achieve perfect order. As shown in Fig. 4(c), it may be possible for the top surface to be covered with bridge structures that from the top appear as unorganized lamellae while the overall quality of the assembly within the film is quite high. Because of the differences in probing depths, the SAXS technique would reflect order before the SEM measurements.

The time required for block copolymers to reach equilibrium on the chemical nanopattern is dependent on many factors including the block copolymer and homopolymer molecular weights, the amount of homopolymer in the blend, and the temperature of annealing. The annealing kinetics are also dependent on factors associated with the chemical pattern, such as the commensurability between $L_{0}$ and $L_{S}$ and the surface energy contrast of neighboring stripes in the chemical pattern. Varying either the commensurability of $L_{0}$ with $L_{S}$ or the contrast in surface energy in the chemical pattern will affect the process latitude for pattern perfection. ${ }^{8,39}$ The time required to achieve perfect assembly is also highly dependent on temperature. ${ }^{12}$ At temperatures above $220^{\circ} \mathrm{C}$, the assembly process takes only a few minutes, although the time required increases for higher molecular weight block copolymers and blends. We previously studied high temperature annealing as a means to achieve very short annealing times. ${ }^{12}$ By annealing at the relatively high temperature of $250{ }^{\circ} \mathrm{C}$, we were able to assemble structures in $104 \mathrm{~kg} / \mathrm{mol}$ PS- $b$-PMMA in $1 \mathrm{~min}$. For a ternary blend from the same $M_{n}$ block copolymer we found the annealing process to take closer to $2 \mathrm{~min}$. Because the annealing time is related to molecular diffusion and the self-diffusion constant of a polymer decreases exponentially with molecular weight, ${ }^{41}$ the time required for annealing increases exponentially with molecular weight. The results with the $176 \mathrm{~kg} / \mathrm{mol}$ blend, shown in Fig. 2, indicate that a longer time was indeed required for perfect assembly with this higher molecular weight blend.

\section{CONCLUSION}

We have shown the feasibility of using transmission SAXS for probing the formation of structure in real time throughout a thin film of a block copolymer blend on a silicon wafer. For ternary blends assembling on a chemical pattern we have verified the formation of various complex intermediate structures and the time frames associated with these structures. The observed ternary blend structures were generally consistent with those reported previously for pure block copolymers, although some new structures were discovered that are likely unique to block copolymer blends. The understanding of the time frame associated with the for- 
mation of these structures should assist in the implementation of block copolymer blends as templates for advanced lithography. Future work with SAXS will focus on increasing the number of detected grating peaks. We plan to use SAXS for studying the kinetics of assembly of block copolymer films on chemical and topographical patterns with more complex pattern shapes that are of high interest to the semiconductor industry.

\section{ACKNOWLEDGMENTS}

The authors would like to thank S. P. Delcambre for his help with the SAXS experiments and data analysis, S. Ji for the brush polymer synthesis, and G. S. W. Craig for help with the manuscript preparation. The XIL patterning and cSAXS measurements were performed at the Swiss Light Source, Paul Scherrer Institut, Villigen, Switzerland. The research was supported by the National Science Foundation Nanoscale Science and Engineering Center at the University of Wisconsin (Grant No. DMR0425880) and by the Semiconductor Research Corporation (Grant Nos. 2005-OC-985 and 2008-OJ-1674).

${ }^{1}$ K. C. Daoulas, M. Muller, M. P. Stoykovich, H. Kang, J. J. de Pablo, and P. F. Nealey, Langmuir 24, 1284 (2008)

${ }^{2}$ E. W. Edwards, M. Muller, M. P. Stoykovich, H. H. Solak, J. J. de Pablo, and P. F. Nealey, Macromolecules 40, 90 (2007).

${ }^{3}$ D. J. C. Herr, Future Fab International, edited by B. Dustrud (Montgomery Research Inc., San Francisco, 2005), p. 18; available at http:// www, future-fab.com.

${ }^{4}$ D. J. C. Herr, Future Fab International, edited by B. Dustrud (Montgomery Research Inc., San Francisco, 2006), p. 20; available at http:// www,future-fab.com.

${ }^{5}$ M. P. Stoykovich and P. F. Nealey, Mater. Today 9, 20 (2006).

${ }^{6}$ International Technology Roadmap for Semiconductors (ITRS) (2007); available at http://www.itrs.net.

${ }^{7}$ M. P. Stoykovich, M. Muller, S. O. Kim, H. H. Solak, E. W. Edwards, J. J. de Pablo, and P. F. Nealey, Science 308, 1442 (2005).

${ }^{8}$ E. W. Edwards, M. F. Montague, H. H. Solak, C. J. Hawker, and P. F. Nealey, Adv. Mater. (Weinheim, Ger.) 16, 1315 (2004).

${ }^{9}$ M. P. Stoykovich, H. Kang, K. C. Daoulas, G. Liu, C. C. Liu, J. J. de Pablo, M. Mueller, and P. F. Nealey, ACS Nano 1, 168 (2007).

${ }^{10}$ R. Ruiz, H. M. Kang, F. A. Detcheverry, E. Dobisz, D. S. Kercher, T. R. Albrecht, J. J. de Pablo, and P. F. Nealey, Science 321, 936 (2008).

${ }^{11}$ C. T. Black, ACS Nano 1, 147 (2007).

${ }^{12}$ A. M. Welander, H. Kang, K. O. Stuen, H. H. Solak, M. Muller, J. J. De Pablo, and P. F. Nealey, Macromolecules 48, 2759 (2008).
${ }^{13}$ J. Holoubek, J. Baldrian, and J. Lal, Chem. Phys. 208, 739 (2007).

${ }^{14}$ J. Holoubek, J. Baldrian, F. Lednicky, S. Malkova, and J. Lal, Macromol. Chem. Phys. 207, 1834 (2006).

${ }^{15}$ J. K. Kim, H. H. Lee, M. Ree, K. B. Lee, and Y. Park, Macromol. Chem. Phys. 199, 641 (1998).

${ }^{16}$ N. Sakamoto and T. Hashimoto, Macromolecules 31, 3815 (1998).

${ }^{17}$ N. Sakamoto and T. Hashimoto, Macromolecules 31, 3292 (1998).

${ }^{18}$ N. Sota, N. Sakamoto, K. Saijo, and T. Hashimoto, Polymer 47, 3636 (2006).

${ }^{19}$ L. Tsarkova, A. Horvat, G. Krausch, A. V. Zvelindovsky, G. J. A. Sevink, and R. Magerle, Langmuir 22, 8089 (2006).

${ }^{20}$ I. W. Hamley, V. Castelletto, Z. B. Lu, C. T. Imrie, T. Itoh, and M. Al-Hussein, Macromolecules 37, 4798 (2004).

${ }^{21}$ C. Harrison, D. H. Adamson, Z. D. Cheng, J. M. Sebastian, S. Sethuraman, D. A. Huse, R. A. Register, and P. M. Chaikin, Science 290, 1558 (2000).

${ }^{22}$ C. Harrison et al., Europhys. Lett. 67, 800 (2004).

${ }^{23}$ C. Harrison et al., Phys. Rev. E 66, 011706 (2002).

${ }^{24}$ A. M. Mayes, T. P. Russell, P. Bassereau, S. M. Baker, and G. S. Smith, Macromolecules 27, 749 (1994).

${ }^{25}$ J. Y. Cheng, C. A. Ross, E. L. Thomas, H. I. Smith, and G. J. Vancso, Appl. Phys. Lett. 81, 3657 (2002).

${ }^{26}$ K. W. Guarini, C. T. Black, and S. H. I. Yeuing, Adv. Mater. (Weinheim, Ger.) 14, 1290 (2002).

${ }^{27}$ R. Ruiz, N. Ruiz, Y. Zhang, R. L. Sandstrom, and C. T. Black, Adv. Mater. (Weinheim, Ger.) 19, 2157 (2007).

${ }^{28}$ R. Ruiz, R. L. Sandstrom, and C. T. Black, Adv. Mater. (Weinheim, Ger.) 19, 587 (2007).

${ }^{29}$ E. W. Edwards, M. P. Stoykovich, M. Muller, H. H. Solak, J. J. De Pablo, and P. F. Nealey, J. Polym. Sci., Part B: Polym. Phys. 43, 3444 (2005).

${ }^{30}$ S. O. Kim, H. H. Solak, M. P. Stoykovich, N. J. Ferrier, J. J. de Pablo, and P. F. Nealey, Nature (London) 424, 411 (2003).

${ }^{31}$ H. Dohi, H. Kimura, M. Kotani, T. Kaneko, T. Kitaoka, T. Nishi, and H. Jinnai, Polym. J. (Tokyo, Jpn.) 39, 749 (2007).

${ }^{32}$ R. Levicky, N. Koneripalli, M. Tirrell, J. F. Ankner, H. Kaiser, and S. K. Satija, Macromolecules 31, 4908 (1998).

${ }^{33}$ V. R. Tirumala et al., Chem. Mater. 19, 5868 (2007)

${ }^{34}$ K. I. Niihara, U. Matsuwaki, N. Torikai, H. Atarashi, K. Tanaka, and H. Jinnai, Macromolecules 40, 6940 (2007).

${ }^{35}$ M. Kato, T. Ito, Y. Aoyama, K. Sawa, T. Kaneko, N. Kawase, and H. Jinnai, J. Polym. Sci., Part B: Polym. Phys. 45, 677 (2007).

${ }^{36}$ H. Sugihara, K. Oya, H. Murase, K. Akabori, K. Tanaka, T. Kajiyama, and A. Takahara, Appl. Surf. Sci. 254, 3180 (2008).

${ }^{37}$ K. Matyjaszewski and J. H. Xia, Chem. Rev. (Washington, D.C.) 101, 2921 (2001).

${ }^{38}$ H. H. Solak, C. David, J. Gobrecht, V. Golovkina, F. Cerrina, S. O. Kim, and P. F. Nealey, Microelectron. Eng. 67-68, 56 (2003).

${ }^{39}$ K. O. Stuen, I. In, E. Han, J. A. Streifer, R. J. Hamers, P. F. Nealey, and P. Gopalan, J. Vac. Sci. Technol. B 25, 1958 (2007).

${ }^{40}$ R. D. Peters, X. M. Yang, Q. Wang, J. J. de Pablo, and P. F. Nealey, J. Vac. Sci. Technol. B 18, 3530 (2000).

${ }^{41}$ H. Yokoyama and E. J. Kramer, Macromolecules 31, 7871 (1998). 